\title{
Physical Modeling of Catalyst Degradation in Low Temperature Fuel Cells: Platinum Oxidation, Dissolution, Particle Growth and Platinum Band Formation
}

\author{
Thomas Jahnke, ${ }^{1, \mathrm{z}}$ Georg A. Futter, ${ }^{1}$ Andrea Baricci, ${ }^{2}$ Claudio Rabissi, ${ }^{2}$ \\ and Andrea Casalegno ${ }^{2, *}$ \\ ${ }^{I}$ German Aerospace Center (DLR), Institute of Engineering Thermodynamics, Computational Electrochemistry, 70569 \\ Stuttgart, Germany \\ ${ }^{2}$ Politecnico di Milano, Dipartimento di Energia, 20156 Milano, Italy
}

\begin{abstract}
The loss of electrochemical active surface area (ECSA) at the cathode is one of the main causes of performance degradation in Polymer Electrolyte Membrane Fuel Cells (PEMFCs). In order to investigate the catalyst degradation and the influence of the operating conditions we develop a multiscale degradation model which includes the formation and reduction of platinum oxides, platinum dissolution, particle growth due to Ostwald ripening, platinum ion transport through the ionomer and platinum band formation in the membrane. This degradation model is coupled with a 2D PEMFC performance model and predictions regarding ion concentration, ECSA evolution and particle growth are validated with dedicated experiments and literature data. Degradation under several AST protocols and under steady state operation are compared and discussed. The importance of a spatially resolved catalyst degradation model is conveyed by the occurrence of a depletion zone in the catalyst layer close to the membrane due to the platinum migration into the membrane. By comparing the correlation between platinum mass loss in the catalyst layer and the ECSA loss we conclude that catalyst degradation under AST conditions with nitrogen is not representative for the degradation under normal operation.

(C) The Author(s) 2019. Published by ECS. This is an open access article distributed under the terms of the Creative Commons Attribution 4.0 License (CC BY, http://creativecommons.org/licenses/by/4.0/), which permits unrestricted reuse of the work in any medium, provided the original work is properly cited. [DOI: 10.1149/2.0232001JES]

(cc) BY
\end{abstract}

Manuscript submitted August 5, 2019; revised manuscript received October 25, 2019. Published November 27, 2019. This paper is part of the JES Focus Issue on Mathematical Modeling of Electrochemical Systems at Multiple Scales in Honor of Richard Alkire.

PEMFCs are a promising alternative to conventional combustion engines in automotive applications due to their zero $\mathrm{CO}_{2}$ emission and the advantages of longer range and fast refueling compared to electric vehicles powered by batteries. Recently, several companies have launched PEMFC powered vehicles. ${ }^{1-4}$ However, the main remaining challenges concern the lifetime and high cost of the PEMFC. ${ }^{5}$ Both aspects are closely related to the platinum catalyst which significantly contributes to the overall cost and whose degradation over time is one of the main causes of performance losses. In order to increase the electrochemically active surface area (ECSA) and therefore the mass activity, the PEMFC catalyst typically consists of platinum or platinum-alloy nanoparticles. However, these particles are not stable under the harsh conditions which can occur in PEMFC operation. ${ }^{6-8}$ In particular, at high potentials platinum dissolution occurs. Since the surface energy is proportional to the inverse of the particle radius, the dissolution rate strongly increases with decreasing platinum particle size. Furthermore, the dissolution rate depends on the platinum oxide coverage. This significantly increases the level of complexity since the dissolution rate will not only depend on the current local conditions but also on it's history which determine the actual oxide coverage. This is of particular importance if one wants to understand the dissolution under highly transient operation such as potential cycling which is often used as an accelerated stress test for the catalyst. ${ }^{9}$ It has been observed that dissolution occurs at both anodic and cathodic sweep of potential cycling. ${ }^{10,11}$ This can be attributed to two different dissolution mechanisms. First, during a fast sweep from low to high potential the platinum oxide layer cannot be formed fast enough to protect the platinum surface from dissolution as it would be the case during steady state operation. Thus, enhanced dissolution is obtained in the high potential region. Second, during the cathodic sweep the platinum oxides are getting reduced where corresponding structural changes also induce platinum dissolution. Since the surface energy of the platinum particles is proportional to the inverse of their size according to the Kelvin equation, ${ }^{12}$ small particles are more prone to dissolution while platinum ion deposition preferably occurs at the larger particles. This leads to the so-called Ostwald-ripening mechanism in which larger

\footnotetext{
*Electrochemical Society Member.
}

${ }^{\mathrm{z} E-m a i l: ~ t h o m a s . j a h n k e @ d l r . d e ~}$ particles grow due to the dissolution of the smaller ones. In addition, dissolved platinum ions can precipitate into the membrane where they form a so-called platinum band. ${ }^{13}$ Both mechanisms lead to a ECSA loss at the cathode side and therefore to performance degradation.

Several models have been developed in the past to describe one or several of the mechanisms described above. ${ }^{14}$ Darling and Meyers ${ }^{12}$ developed a model for platinum dissolution coupled with a kinetics for a single platinum oxide species to study the dependence of platinum dissolution on cell potential and particle radius. Only a single particle radius was considered in this work so Ostwald-ripening was not included. Later, the same authors developed a model which considers the transport of platinum ions in the ionomer taking into account particles of two distinct sizes. ${ }^{15}$ The platinum band formation was not included in this work. Bi et al. ${ }^{13}$ proposed a simple model to predict the position of the platinum band which was in agreement with their experimental observations. The platinum dissolution itself was not part of the model. Holby et al. ${ }^{16}$ presented a 1D model which described the Ostwald ripening by means of the evolution of the particle size distribution (PSD). They also included a sink term for the platinum ions at a fixed position inside the membrane to describe the effect of the platinum band formation without taking into account the fluxes of hydrogen and oxygen. Later Holby and Morgan ${ }^{17}$ further improved the model and studied the effect of PSD and position of the platinum band on the degradation. Rinaldo et al. ${ }^{18}$ presented a model which included platinum dissolution and platinum oxide dissolution. By fitting the model to experimental data for different degradation tests, they observed effective surface tensions and rate constants for the dissolution, depending on the operating conditions. The effect of platinum band formation was not taken into account in this study. A detailed model for the platinum band formation was proposed by Burlatsky et al. ${ }^{19}$ The model includes the nucleation of the platinum particles as well as the consequent particle growth. Ahluwalia et al. ${ }^{20}$ developed a model for platinum dissolution to investigate the effect of potential, particle size and oxide coverage. In a later work Ahluwalia et al. ${ }^{21}$ proposed a model which includes platinum dissolution, redeposition and coalescence. The model was validated under aqueous tests at room temperature as well as under AST conditions. Recently, Baroody et al..$^{22}$ have presented a physical-statistical OD model for the platinum degradation based on a previous model developed in the same group ${ }^{23}$ which takes into account dissolution and redeposition as well as coagulation, 
particle detachment and the loss of platinum in the membrane. Effective kinetic parameters for the different mechanisms were obtained by fitting the model to experimental data sets taken from literature. It was found that in case of medium surface carbon supports dissolution, redeposition and platinum loss in the membrane give the main contribution to total ECSA loss for potential cycles with $1.0 \mathrm{~V}$ upper potential limit. Instead, for low surface carbon also a contribution due to coagulation was obtained. At higher potential an additional contribution due to particle detachment was obtained which was attributed to carbon corrosion. Also recently, a detailed model for the oxygen reduction and oxide growth has been presented by Jayasankar and Karan. ${ }^{24}$ The kinetic multi-step model was validated against cyclic voltammetry and potentiostatic oxide growth measurements. Very recently, Schneider et al. ${ }^{25}$ developed a zero-dimensional catalyst degradation model which includes platinum oxidation, place-exchange, dissolution, Ostwald ripening as well as platinum loss due to carbon corrosion and platinum ion diffusion into the membrane. The model was validated with ECSA measurements for various operating conditions and the contribution of the various mechanisms on the overall ECSA loss was investigated.

To summarize, a lot of work has been done in the past to model the catalyst degradation in PEMFC, in particular by developing detailed models on certain mechanisms or zero-dimensional models of combined mechanisms. However, to the best of the authors knowledge a model which is able to predict the spatially resolved catalyst degradation under various operating conditions including highly transient AST protocols is not yet available. To achieve this goal the coupling between a spatially resolved performance model and a degradation model which accurately describes all relevant mechanisms from the sub-nanometer scale up to the cell level is required. On the other hand the model should be kept as simple as possible to be still solvable with acceptable computational costs. By coupling novel submodels for platinum oxidation, platinum dissolution, Ostwald-ripening and platinum band formation with a $2 \mathrm{D}$ single cell performance model the degradation model presented in this manuscript is capable of accurately describing the catalyst degradation under steady-state and transient operation with a single set of parameters. This model allows investigating the effect of different AST protocols on catalyst degradation and provides insight on the differences between the degradation under AST and real world operation. Furthermore, the spatial resolution of the model allows investigations on the occurring heterogeneities in catalyst degradation.

This paper is organized as follows. In the Physical model section we introduce the physical degradation model. In the Coupling with the cell model section we describe how the degradation model is coupled with the physical 2D PEMFC model in our modeling framework NEOPARD-X. ${ }^{26,27}$ In the Experimental section we describe the experiments performed for model validation. The results consisting of model predictions and validation are presented in the Results section. A conclusion and summary of the main results can be found in the Conclusions section.

\section{Physical Model}

In this section we introduce the physical model describing the catalyst degradation. The multiscale model includes platinum oxidation and reduction, platinum dissolution, particle growth and platinum band formation which are described in the following subsections. The corresponding model parameters are listed in Table I. This set of parameters is used for all simulations presented in this work.

Platinum oxide model.-Platinum oxidation plays an important role for the catalyst degradation as it strongly affects the platinum dissolution mechanism. In particular, when considering transient operation like fast potential cycling, an accurate description of the oxide evolution is thus needed for modeling the degradation. Dedicated experiments show a strong dependence of oxide coverage on cathode potential and a logarithmic increase of this coverage/charge with time (cf. Platinum oxide coverage section). This logarithmic growth of the oxide coverage has also been observed by Conway and co-workers
Table I. Model parameters.

Symbol/Value

Units

Comment

Platinum oxide:

$k_{2}=8.75 \times 10^{-5}$ $k_{1}=50.0$

$\omega_{1}=1.1 \times 10^{4}$

$\alpha_{1}=0.5$

$E_{01}=0.75$

$\omega_{2}=1.3 \times 10^{5}$

$\alpha_{2}=0.25$

$k_{3}=2.8 \times 10^{18}$

$\alpha_{3}=2.0$

Platinum dissolution:

$k_{f}=1.14 \times 10^{9}$

$k_{r}=3.1 \times 10^{6}$

$E_{0, d 1}=6.39 \times 10^{4}$

$n=2$

$\alpha=0.3$

$\gamma_{0}=2.4$

$\Gamma=2.1 / \mathrm{F}$

$k_{f, 2}=3 \times 10^{-2}$

Ostwald ripening:

$u_{\mathrm{Pt}^{2+}}^{0}=3 \times 10^{-13}$

Platinum-band formation:

$k_{\text {Pt-band }}=1 \times 10^{5}$

$k_{\mathrm{H} 2 \mathrm{c}}=1 \times 10^{7}$
$\Omega=9.09 \times 10^{-6}$

$\mathrm{s}^{-1}$
$\mathrm{~J} \mathrm{~mol}^{-1}$
-
$\mathrm{V}$
$\mathrm{s}^{-1}$
$\mathrm{~J} \mathrm{~mol}^{-1}$
-
$\mathrm{s}^{-1}$$$
\mathrm{s}^{-1}
$$$$
\mathrm{m}^{3} \mathrm{~mol}^{-1} \mathrm{~s}^{-1}
$$$$
\mathrm{J} \mathrm{mol}^{-1}
$$$$
\text { - }
$$$$
-
$$$$
\mathrm{J} \mathrm{m}^{-2}
$$$$
\text { mol m }{ }^{-2}
$$$$
\mathrm{m}^{3} \mathrm{~mol}^{-1}
$$$$
\text { - }
$$$$
\mathrm{s} \text { mol kg } \mathrm{kg}^{-1}
$$

fitted

fitted

assumed

fitted

fitted

fitted

fitted

fitted

fitted

fitted

fitted

17

assumed

fitted

17

29

17

fitted

assumed

$\mathrm{m}^{3} \mathrm{~mol}^{-1} \mathrm{~s}^{-1}$

$\mathrm{m}^{9} \mathrm{~mol}^{-3} \mathrm{~s}^{-1}$

assumed

assumed who attributed this effect to a slow place exchange between the oxygen and platinum atoms. ${ }^{28}$ The authors proposed a three-step mechanism consisting of one fast initial oxidation step followed by a slow irreversible place exchange and an irreversible reduction of the oxides. However, this model was not specified in terms of the reaction kinetics for these three steps. Based on our experimental data, we propose a slightly different semi-empirical three-step mechanism for the platinum oxidation, including two charge-transfer reactions as described in the following. First, a fast reversible oxidation step

$$
\mathrm{Pt}+\mathrm{H}_{2} \mathrm{O} \leftrightarrow \mathrm{PtOH}+\mathrm{H}^{+}+\mathrm{e}^{-}
$$

followed by a second, slower, irreversible oxidation step

$$
\mathrm{PtOH} \longrightarrow \mathrm{PtO}+\mathrm{H}^{+}+\mathrm{e}^{-}
$$

and an irreversible reduction step

$$
\mathrm{PtO}+2 \mathrm{H}^{+}+2 \mathrm{e}^{-} \longrightarrow \mathrm{Pt}+\mathrm{H}_{2} \mathrm{O}
$$

The place exchange is not explicitly modeled but is implicitly reflected by the irreversible formulation of this semi-empirical model. The kinetics of these reactions is described by

$$
\begin{gathered}
r_{1}=k_{1}\left[\left(1-\theta_{\mathrm{PtOH}}\right) a_{\mathrm{H}_{2} \mathrm{O}} e^{-\frac{\omega_{1} \theta_{\mathrm{PtOH}}}{\mathrm{R} T}} e^{\frac{\alpha_{1} \mathrm{~F}}{\mathrm{R} T}\left(\Delta \phi-E_{01}\right)}\right. \\
\left.-\theta_{\mathrm{PtOH}} a_{\mathrm{H}^{+}} e^{-\frac{\left(1-\alpha_{1}\right) \mathrm{F}}{\mathrm{RT}}\left(\Delta \phi-E_{01}\right)}\right], \\
r_{2}=k_{2} \theta_{\mathrm{PtOH}} e^{-\frac{\omega_{2} \theta_{2}}{\mathrm{RT}}} e^{\frac{\alpha_{2} \mathrm{~F}}{\mathrm{R} T} \Delta \phi}, \\
r_{3}=k_{3} \theta_{\mathrm{PtO}} a_{\mathrm{H}^{+}}^{2} e^{-\frac{\alpha_{3} \mathrm{~F}}{\mathrm{RT}} \Delta \phi} .
\end{gathered}
$$

The surface coverages are calculated as

$$
\begin{aligned}
\frac{d \theta_{\mathrm{PtOH}}}{d t} & =r_{1}-r_{2}, \\
\frac{d \theta_{\mathrm{PtO}}}{d t} & =r_{2}-r_{3} .
\end{aligned}
$$


The corresponding source terms for the ionic and electronic charge balance equations are

$$
q_{\mathrm{H}^{+}}=-q_{\mathrm{e}^{-}}=\left(r_{1}+r_{2}-2 r_{3}\right) \times E C S A \times 2.1 \frac{C}{\mathrm{~m}_{\mathrm{Pt}}^{2}} .
$$

As discussed in the Platinum oxide coverage section this model is capable of describing the experimentally observed potential dependent oxide coverage evolution with high precision. The kinetic parameters for Reactions 4-6 have been fitted based on the experimental data presented in Fig. 1.

Platinum dissolution.-Two mechanisms are considered for the platinum dissolution. ${ }^{10,12}$ Direct dissolution of platinum according to

$$
\mathrm{Pt} \leftrightarrow \mathrm{Pt}^{2+}+2 \mathrm{e}^{-},
$$

and dissolution occurring due to the reduction of platinum oxides

$$
\mathrm{PtO}+2 \mathrm{H}^{+} \longrightarrow \mathrm{Pt}^{2+}+\mathrm{H}_{2} \mathrm{O} \text {. }
$$

For the first mechanism the dissolution rate strongly depends on the particle size as the surface energy is given by ${ }^{16,18}$

$$
\Delta \mu=\mu(r)-\mu(\infty)=\frac{2 \Omega \gamma(\theta)}{r} .
$$

Here, $\Omega$ denotes the molar volume of platinum, $\gamma$ is the surface tension and $r$ the particle radius. The surface tension depends on the coverage of the platinum oxides. Based on the platinum oxide model introduced in the Platinum oxide model section this dependence can be derived (cf. Appendix A). We obtain

$$
\begin{aligned}
\gamma(\theta)= & \gamma_{0}+\Gamma \mathrm{R} T\left[\theta_{\mathrm{PtOH}} \log \left(\theta_{\mathrm{PtOH}}\right)+\left(1-\theta_{\mathrm{PtOH}}\right) \log \left(1-\theta_{\mathrm{PtOH}}\right)\right. \\
& -\frac{\mathrm{F}}{\mathrm{R} T}\left(\Delta \phi-E_{01}\right) \theta_{\mathrm{PtOH}}+\frac{\omega_{1} \theta_{\mathrm{PtOH}}^{2}}{2 \mathrm{R} T}+\theta_{\mathrm{PtO}} \log \left(\theta_{\mathrm{PtO}}\right)-\theta_{\mathrm{PtO}} \\
& \left.+\log \left(\frac{k_{3}}{k_{2} \theta_{\mathrm{PtOH}}}\right) \theta_{\mathrm{PtO}}-\frac{\left(\alpha_{2}+\alpha_{3}\right) \mathrm{F}}{\mathrm{R} T} \Delta \phi \theta_{\mathrm{PtO}}+\frac{\omega_{2} \theta_{\mathrm{PtO}}^{2}}{2 \mathrm{R} T}\right] .
\end{aligned}
$$

With this surface tension the rate of the first dissolution and redeposition mechanism 10 for particles with radius $r$ is calculated as

$$
\begin{aligned}
r_{\mathrm{Pt}, 1}(r)= & A(r) \Gamma k_{f} e^{\frac{-E_{a}}{\mathrm{R} T}}\left[\left(1-\theta_{\mathrm{PtOH}}\right) e^{\frac{(1-\alpha) n \mathrm{~F}}{\mathrm{R} T}\left(\Delta \phi-E_{0, d 1}\right)} e^{\frac{(1-\alpha) 2 \Omega \gamma}{\mathrm{R} T r}}\right. \\
& \left.-\frac{k_{r}}{k_{f}} c_{\mathrm{Pt}^{2+}} e^{\frac{-\alpha n \mathrm{~F}}{\mathrm{R} T}\left(\Delta \phi-E_{0, d 1}\right)} e^{-\frac{\alpha 2 \Omega \gamma}{\mathrm{R} T r}}\right],
\end{aligned}
$$

where $A(r)$ denotes the surface area of all particles with radius $r$ and $c_{P t^{2+}}$ is the local platinum ion concentration. The kinetic parameter $k_{f}$ mainly determines the degradation rate during steady state operation while under transient operation the degradation rate can vary significantly due to changes in the oxide coverage dependent surface tension 13. The kinetics $k_{r}$ of the backward reaction, i.e., the redeposition determines the equilibrium platinum ion concentration and was validated with experimental data from literature as discussed in the Potential dependent ion concentration section.

The idea behind the second mechanism 11 is that the platinum oxidation can lead to a place exchange between the platinum and oxygen atoms, which exposes the oxidized platinum to the electrolyte..$^{10} \mathrm{Re}$ duction of the oxide then might cause a dissolution of these platinum atoms. As we will see, this second mechanism leads to a dissolution during cathodic sweeps in potential cycles, which has been observed experimentally in the group of Mayrhofer. ${ }^{10,11}$ We assume the kinetics of the second mechanism to be dependent on the number of atoms at the edges of the platinum particles, which should be more prone to dissolution. The rate of the second dissolution mechanism is thus defined as

$$
r_{\mathrm{Pt}, 2}(r)=A(r) \Gamma k_{f, 2} X_{\text {edge }}(r) r_{3},
$$

where $r_{3}$ is the platinum oxide reduction rate defined in Eq. 6. $X_{\text {edge }}$ describes the fraction of edge sites of the platinum particle. This value depends on the size and geometry of the particles. Even though arbitrary particle geometries could be considered in principle, for simplicity we restrict ourselves to the cuboctahedron type proposed by Redmond et al. ${ }^{29}$ In this case, $X_{\text {edge }}$ is calculated according to

$$
X_{\text {edge }}=\frac{12+24\left(n_{\text {edge }}-2\right)}{6\left(n_{\text {edge }}-2\right)^{2}+4\left(n_{\text {edge }}-3\right)\left(n_{\text {edge }}-2\right)+12+24\left(n_{\text {edge }}-2\right)},
$$

with the number of atoms along the edge given by

$$
n_{\text {edge }}=\left[\frac{4 \pi}{\sqrt{50}}\right]^{1 / 3} \frac{r}{2.77 \cdot 10^{-10}},
$$

To ensure that our results are not strongly affected by this particular choice of particle geometry, we made a similar calculation for the truncated octahedron, which has been shown to be the most stable geometry for platinum particles. ${ }^{30}$ The calculation and a figure with the comparison of both geometries can be found in the supplementary material. We obtain that the dependence of $X_{\text {edge }}$ on the particle radius is indeed very similar for both cases. The second dissolution mechanism becomes important in particular during cycling in a potential range in which platinum oxides are frequently formed and reduced.

Ostwald ripening.-As we have discussed in the Platinum dissolution section, the platinum dissolution rate strongly depends on the size of the platinum particles: the smaller the particles the faster the dissolution. Fuel cell catalysts consist of particles with different sizes, which can be described by a particle size distribution $N(r)$, i.e., the number of particles of radius $r$ per catalyst volume. Since the dissolution rate depends on the particle size, the local ion concentration will deviate from the equilibrium concentration for a given particle radius $r$, which could be calculated from Eq. 14 by setting $r_{\text {diss }, 1}=0$. For small particles the equilibrium ion concentration will be higher than the local ion concentration causing dissolution, while for larger particles the ion concentration will be larger than their equilibrium value causing precipitation of the ions on the particles. Overall this leads to a growth of the average particle size, known as Ostwald ripening. ${ }^{31}$ The change of radius of a particle at given size can be calculated from the total dissolution rate $r_{\mathrm{Pt}}=r_{\mathrm{Pt}, 1}+r_{\mathrm{Pt}, 2}$ as

$$
\left.\frac{d r}{d t}\right|_{\text {Ostwald }}=-\frac{\Omega}{A(r)} r_{\mathrm{Pt}}(r)
$$

where $\Omega$ is the molar volume of platinum and $A(r)$ is the surface area of all particles with radius $r$. This leads to the balance equation for the particle size distribution

$$
\frac{d N(r)}{d t}+\frac{d}{d r}\left(\left.N(r, t) \frac{d r}{d t}\right|_{\text {Ostwald }}\right)=0 .
$$

Note, that in general this balance equation could also include additional terms for particle detachment ${ }^{22}$ or coagulation ${ }^{21,22}$ which are assumed to be negligible here. Since a good agreement with both experimental PSD and ECSA evolution is obtained (cf. ECSA loss and particle growth in Direct Methanol Fuel Cell section) we conclude that this assumption is justified. This simplification reduces the the numerical complexity significantly as coagulation would require to solve integrodifferential equations.

The change of local ion concentration due to Ostwald ripening is calculated by integrating the dissolution/precipitation rates over all particle radii

$$
q_{\mathrm{Pt}^{2+}, \text { Ostwald }}=\int_{0}^{\infty} N(r) r_{\mathrm{Pt}}(r) d r=-\left.\frac{4 \pi}{3 \Omega} \int_{0}^{\infty} r^{3} \frac{d N}{d t}\right|_{\text {Ostwald }} d r .
$$

The platinum ions can move within the ionomer phase due to gradients in the concentration and ionomer potential according to the NernstPlanck equation

$$
\frac{d\left(\phi_{\text {ionomer }} c_{\mathrm{Pt}^{2+}}\right)}{d t}+\nabla \cdot \Phi_{\mathrm{Pt}^{2+}}-q_{\mathrm{Pt}^{2+}}=0
$$


with

$$
\Phi_{\mathrm{Pt}^{2+}}=-u_{\mathrm{Pt}^{2+}} \mathrm{R} T \nabla c_{\mathrm{Pt}^{2+}}-2 F u_{\mathrm{Pt}^{2+}} c_{\mathrm{Pt}^{2+}} \nabla \Phi_{\text {ion }},
$$

where the mobility of the platinum ions is assumed to be proportional to the water volume fraction $f(\lambda)$ in the ionomer as proposed by $\mathrm{Bi}$ et al., ${ }^{32}$ i.e.,

$$
u_{\mathrm{Pt}^{2+}}=u_{\mathrm{Pt}^{2+}}^{0} f(\lambda)
$$

with

$$
f(\lambda)=\frac{\lambda V_{\mathrm{H}_{2} \mathrm{O}}}{V_{\mathrm{mem}}+\lambda V_{\mathrm{H}_{2} \mathrm{O}}},
$$

where $V_{\mathrm{H}_{2} \mathrm{O}}$ and $V_{\text {mem }}$ are the molar volumes of water and the dry membrane, respectively. The local water content $\lambda$ in the membrane is calculated with the coupled single cell model (cf. Coupling with the cell model section).

In the electrode the source/sink term $q_{\mathrm{Pt}^{2+}}$ is given by Ostwaldripening (Eq. 20) while in the membrane the sink term is due to the platinum band formation as described in the Platinum band formation section.

Platinum band formation.-Platinum ions can move in the ionomer from the electrode into the membrane as described by Eq. 21 . In the membrane they can react with hydrogen coming from the anode side according to

$$
\mathrm{Pt}^{2+}+\mathrm{H}_{2} \leftrightarrow \mathrm{Pt}+2 \mathrm{H}^{+} .
$$

Thus, Reaction 25 represents a sink for the platinum ions in Eq. 21 and the source for the platinum band formation. In principle different levels of detail could be taken into account when modeling the platinum deposition in the membrane, e.g., the platinum particles in the membrane could be described by a particle size distribution in a similar way as done in the catalyst layer. However, this would add a significant amount of complexity and computational cost. In this work we are mainly interested in the amount and position of platinum deposition within the membrane but not in the particle sizes. Therefore, the platinum deposition within the membrane is characterized by the local platinum concentration instead of a particle size distribution. The rate for Reaction 25 is calculated as

$$
r_{\mathrm{Pt} \text {-band }}=k_{\mathrm{Pt} \text {-band }} c_{\mathrm{Pt}^{2+}} c_{\mathrm{H}_{2}} \text {. }
$$

The kinetics of 26 determines the width of the platinum band. The slower the reaction the broader the platinum band. Experimentally observed platinum bands are often very narrow. Helmly et al..$^{33}$ reported a measured platinum band width of about $2.5 \mu \mathrm{m}$. We choose the kinetic parameter $k_{\mathrm{Pt} \text {-band }}=10^{5} \frac{\mathrm{m}^{3}}{\mathrm{~mol}}$, which gives us a similar value for the platinum band thickness.

On the deposited platinum within the membrane, catalytic combustion of hydrogen with oxygen from the cathode can take place according to

$$
2 \mathrm{H}_{2}+\mathrm{O}_{2} \leftrightarrow 2 \mathrm{H}_{2} \mathrm{O} .
$$

The kinetics of this combustion reaction is calculated as

$$
r_{\mathrm{H} 2 \mathrm{c}}=k_{\mathrm{H} 2 \mathrm{c}} c_{\mathrm{Pt}_{\mathrm{t}}} c_{\mathrm{H}_{2}}^{2} c_{\mathrm{O}_{2}} \text {. }
$$

This reaction determines the position of the platinum band due to the consumption of hydrogen which is also needed for the platinum band formation reaction 25 . Under steady state conditions, the platinum band will be formed in a region where the hydrogen and oxygen fluxes compensate each other, i.e., where the hydrogen flux is twice the oxygen flux. We calculate the hydrogen and oxygen permeation via coupling with our single cell model ${ }^{26}$ ( $\mathrm{cf}$. Coupling with the cell model section) based on the model of Weber and Newman ${ }^{34}$ which provides us with the local hydrogen and oxygen concentrations needed to calculate the reaction rates of 26 and 28 . The kinetic parameter $k_{\mathrm{H} 2 \mathrm{c}}$ determines how much of the permeating gases are actually converted to water at the platinum band, i.e., how much the platinum band reduces the gas crossover.
The gas permeation does not only determine the position but also the amount of platinum deposited in the membrane. If the platinum band is formed closer to the cathode, higher gradients in the platinum ion concentration lead to faster ion transport and therefore to an increase of the deposited platinum.

\section{Coupling with the Cell Model}

The presented degradation model is implemented in our modeling framework NEOPARD- $\mathrm{X}^{26,27}$ which is based on Dumu ${ }^{\mathrm{X} 35}$ and is directly coupled with the previously developed single cell PEMFC model. ${ }^{26}$ This model includes

1. A 2D along-the-channel geometry with nine spatially resolved layers (anode and cathode channels, GDLs, MPLs, CLs and the membrane)

2. A multiphase Darcy model for the two-phase, multicomponent transport within the porous electrodes

3. A membrane model including coupled water and proton transport as well as transport of dissolved gas species

4. Proton transport through the ionomer within the CLs and electron transport through the support phase of the porous electrodes

5. Butler-Volmer kinetics for the ORR and HOR reactions

6. An ionomer film model describing the oxygen transport from the gas phase through the ionomer film to the cathode catalyst

7. Energy transport through all layers of the cell

All corresponding model equations are discussed in detail in Ref. 26. The only modification of this single cell model is with respect to the platinum oxide submodel which has been replaced by the new model presented in the Platinum oxide model section.

For the coupling the model equations of the degradation mechanisms are solved locally at each point within the cell, where the local conditions like local potentials, species concentrations, temperature, etc. are obtained from the cell model. In particular, the local water volume fraction in the ionomer needed for the platinum ion mobility and the concentrations of hydrogen and oxygen in the membrane needed for the platinum band model are obtained from this coupling. On the other hand, the degradation model affects the performance model by modifying the local ECSA and therefore cell performance. The local ECSA is obtained from the time dependent local PSD according to

$$
E C S A=\zeta \int_{0}^{\infty} 4 \pi N(r) r^{2} d r,
$$

where $\zeta$ is the catalyst utilization, i.e., the ratio between geometrical surface area of the particles and active surface area. The value of $\zeta$ is assumed to be constant and calculated by Eq. 29 from the initial PSD and initial ECSA measurement.

\section{Experimental}

Materials.-Experimental characterization was carried out on $25 \mathrm{~cm}^{2}$ membrane electrode assembly (MEA) manufactured by EWII Fuel Cells A/S, whose specifications are summarized in the Table II. The platinum loading in both anode and cathode and the thicknesses of the membrane and GDLs represent the state of the art for stationary hydrogen or methanol fuel cells.

Accelerated stress test.-In order to characterize the stability of the electrocatalyst with regard to platinum dissolution and ripening, the AST protocol defined by the U.S Department of Energy was adopted. ${ }^{36}$ According to the protocol, the cathode potential was cycled between $0.6 \mathrm{~V}$ and $1 \mathrm{~V}$ with a sweep rate of $50 \mathrm{mV} \mathrm{s}^{-1}$, following a triangular wave, under fully humidified gas dry flow rate of $0.05 \mathrm{Nl} \mathrm{min}{ }^{-1}$ of $\mathrm{H}_{2}$ and $\mathrm{N}_{2}$ respectively at anode and cathode.

DMFC stress test.-To investigate degradation of the CCL related to DMFC operation, a DMFC specific operation protocol, discussed 
Table II. MEA sample specifications.

\begin{tabular}{|c|c|c|c|c|c|}
\hline & Anode GDL & Anode CL & Membrane & Cathode CL & Cathode GDL \\
\hline PEMFC & SGL 25BC & $0.2 \mathrm{mgPt} \mathrm{cm}{ }^{-2}$ Carbon black & Nafion 212 & $0.6 \mathrm{mgPt} \mathrm{cm}^{-2}$ Graphitized carbon & SGL 25BC \\
\hline DMFC & SGL 35DC & $1.8 \mathrm{mgPtRu} \mathrm{cm}{ }^{-2}$ Carbon black & Nafion 115 & $1.2 \mathrm{mgPt} \mathrm{cm}^{-2}$ Graphitized carbon & SGL 35DC \\
\hline
\end{tabular}

by the authors in Ref. 37, has been analyzed in the present work. During the test, nominal current density and cell operating temperature are $0.25 \mathrm{~A} \mathrm{~cm}^{-2}$ and $75^{\circ} \mathrm{C}$, respectively. Anode and cathode are fed with 1.0 M methanol solution and air saturated with water at ambient temperature, respectively (stoichiometries equal to 6 and 3 respectively at $0.25 \mathrm{~A} \mathrm{~cm}^{-2}$ ). As discussed in ${ }^{37}$ and indicated by the MEA manufacturer, the DMFC reference operating protocol consists of periods of 20 minutes in galvanostatic operation interspersed by 1 minute of refresh cycle procedure. This procedure, thoroughly investigated in Ref. 38, consists of a sequence of OCV and cathode air break and is intended to revert temporary degradation during operation. During the air break, the cathode potential is expected to drop below $0.5 \mathrm{~V}$ to reduce platinum oxides generated during the DMFC high-potential cathode operation. Moreover, as discussed in Ref. 37, a longer full refresh is performed every $100 \mathrm{~h}$ to revert temporary degradation and perform electrochemical diagnostics.

Measurement of ECSA and oxides coverage.-The electrochemical active surface area is measured with cyclic voltammetry under fully

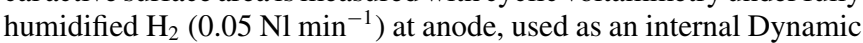
Reference Electrode (DHE), ${ }^{39}$ and $\mathrm{N}_{2}\left(0.05 \mathrm{Nl} \mathrm{min}^{-1}\right)$ at cathode and $80^{\circ} \mathrm{C}$ cell temperature. The ECSA is obtained by integrating the current density peak related to the hydrogen desorption, corrected for a constant double layer current, according to

$$
E C S A=\frac{\int_{E_{\mathrm{OCV}}}^{E_{\mathrm{DL}}} i-i_{\mathrm{DL}} d E}{\sigma_{m} L_{\mathrm{Pt}} s r},
$$

where $E$ is the electrode potential, $s r$ is the scan rate, the minimum potential $\left(E_{\mathrm{OCV}}\right)$ is the open circuit potential measured under $\mathrm{H}_{2} / \mathrm{N}_{2}$ atmosphere and the double layer potential $\left(E_{\mathrm{DL}}\right)$ is set to $0.4 \mathrm{~V}$. It is assumed that the cathode catalyst is covered by a monolayer of hydrogen with a charge density of $\sigma_{m}=2.1 \mathrm{C} \mathrm{m}^{2}$. A protocol was applied to estimate the platinum oxide coverage after potential holding under $\mathrm{H}_{2} / \mathrm{N}_{2}$. The protocol, as in Ref. 40, is based on a linear sweep and a constant potential period at $E_{\max }$, where Pt oxidation occurs, followed by a linear sweep voltammetry from $E_{\max }$ to the minimum potential, which permits to reduce $\mathrm{Pt}$ oxides restoring $\mathrm{Pt}$ metallic form. The charge related to the removal of platinum oxides is calculated by integrating the current in the region of the voltammogram (cf. Fig. 1) between the potential $\left(E_{\max }\right)$ and the double layer potential, indicative of the double layer current. The ratio between integrated oxide area and ECSA is assumed to be the total platinum oxide coverage

$$
\theta_{\mathrm{PtOx}}=\frac{1}{E C S A} \frac{\int_{E_{\mathrm{DL}}}^{E_{\mathrm{hold}}} i-i_{\mathrm{DL}} d E}{n_{e} \sigma_{m} L_{\mathrm{Pt}} s r},
$$

where $\sigma_{m}$ is the stripping charge for a single electron oxide and $n_{e}$ is the number of electrons involved in the PtOx reduction which is set equal to 2 when assuming a $\mathrm{PtO}$ basis for coverage estimation.

TEM and analysis of particle size distribution.--In order to quantify the particle growth related to DMFC operation, a TEM-based particle size distribution (PSD) analysis has been performed on the cathode electrode of pristine and aged MEAs. The PSD analysis is based on TEM imaging performed by $\mathrm{CEA}^{\mathrm{a}}$ in the frame of a former work, ${ }^{37}$ where the procedure and the TEM images are thoroughly

${ }^{a}$ Commissariat à l'énergie atomique et aux énergies alternatives, LITEN. detailed. Each sample was first embedded in epoxy resin and prepared into thin slices $(<90 \mathrm{~nm}$ ) using a LEICA ultramicrotome, then analyzed in BF (bright field) or HAADF/STEM (high angle annular dark field/scanning TEM) mode, on a FEI-Titan Ultimate microscope equipped with a Cs aberration probe corrector.

PSD histograms were calculated based on at least 500-800 particles per region, so to have a statistically valid sample.

Based on the data for the pristine MEA the initial PSD has been fitted by the log-normal distribution

$$
N(r)=\frac{N_{0}}{\sigma_{0} r \sqrt{2 \pi}} e^{\frac{-\left(\log (r)-\log \left(r_{0}\right)\right)^{2}}{2 \sigma_{0}^{2}}},
$$

with $\sigma_{0}=0.31$ and $r_{0}=1.58 \cdot 10^{-9} \mathrm{~m} . N_{0}$ is calculated from the platinum loading $M_{\mathrm{Pt}}$ according to

$$
N_{0}=\frac{M_{\mathrm{Pt}}}{d_{\mathrm{CL}} \rho_{\mathrm{Pt}} \int_{0}^{\infty} N(r) / N_{0} \frac{4}{3} \pi r^{3} d r},
$$

where $d_{\mathrm{CL}}=20 \mu \mathrm{m}$ is the CL thickness and $\rho_{\mathrm{Pt}}=21450 \mathrm{~kg} / \mathrm{m}^{3}$ is the density of platinum. This distribution is used as initial PSD for all the following simulations. The catalyst utilization $\zeta$ is then calculated from the measured initial ECSA and the PSD as

$$
\zeta=\frac{E C S A_{\text {init }}}{N_{0} \int_{0}^{\infty} N(r) 4 \pi r^{2} d r} .
$$

\section{Results}

Ambiguity is an important issue for complex models which contain many unknown parameters. To address this issue several measures are used here for model validation, i.e., the evolution of the platinum oxide coverage, the platinum ion concentration, the ECSA evolution as well as the evolution of the particle size distribution during various degradation tests as discussed in the following. In this way the ambiguity of the model parametrization is reduced and the confidence in accurately describing the degradation mechanisms is increased significantly.

Platinum oxide coverage.-An accurate description of the platinum oxide coverage evolution is important for describing the catalyst degradation since the coverage directly affects the platinum dissolution as described in the Platinum dissolution section. Therefore, the model introduced in the Platinum oxide model section has been validated with the dedicated CV experiments described in the Measurement of ECSA and oxides coverage section. For example, Fig. 1 reports the measured evolution of the platinum oxides reduction peak when varying the potential of the holding period at a fixed duration of $600 \mathrm{~s}$ in a) and when varying the duration of the holding period at a fixed potential of $0.85 \mathrm{~V}$ in b). For validation of the platinum oxide model the cell potential in the simulation is held fixed at various values for 20 minutes and the simulated evolution of the total oxide coverage on $\mathrm{PtO}$-basis $\left(\theta=0.5 \theta_{P t O H}+\theta_{P t O}\right)$ is compared with the one determined from integrating the measured peaks in the respective CVs during cathodic sweep. Fig. 1c shows the comparison between simulated and measured platinum oxide coverages. As one can see, the model accurately describes the observed evolution over the whole range of considered operating conditions. In particular, the model describes the fast initial formation of oxide coverage followed by a logarithmic growth of the coverage with time at high potentials. Instead, at low potentials 


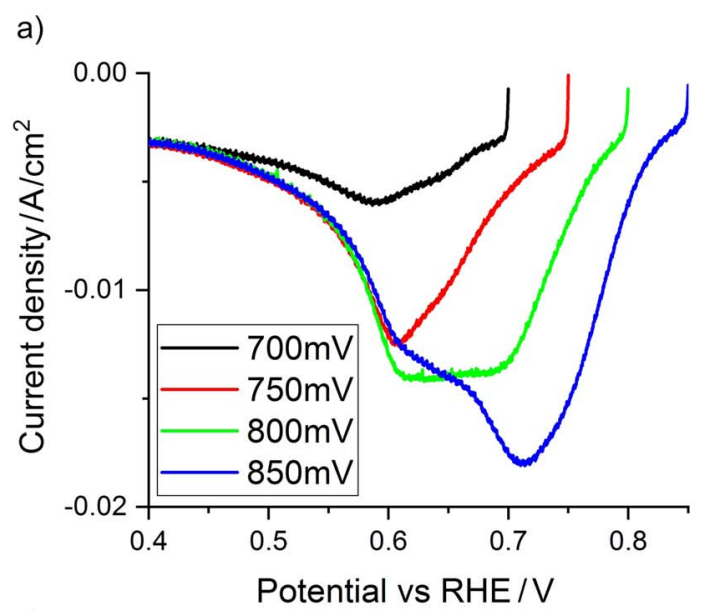

b)

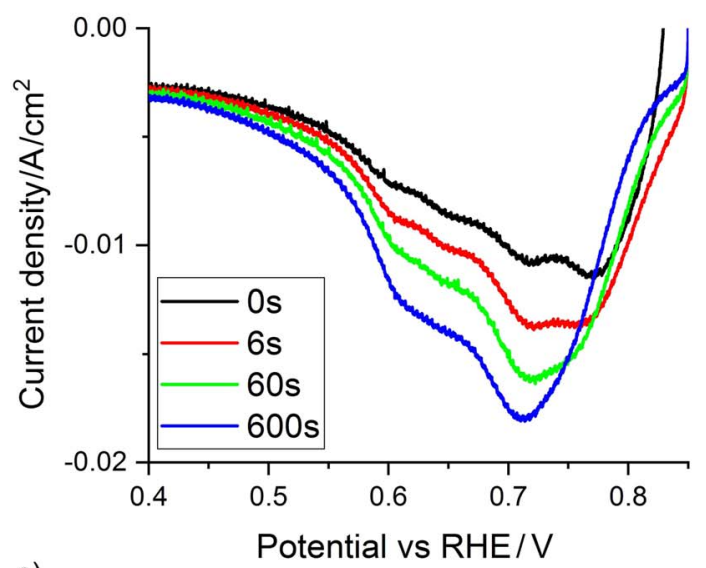

c)

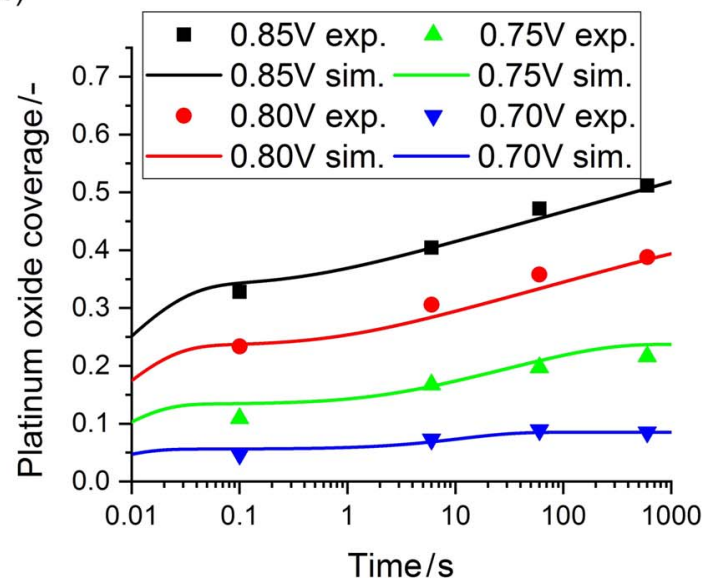

Figure 1. Platinum oxide reduction peaks measured with a) different holding potentials after $600 \mathrm{~s}$ and b) different holding periods at $0.85 \mathrm{~V}$. c) Platinum oxide coverage evolution for various cathode potentials. Symbols represent the experimental values and lines the respective simulations.

a steady state is reached after a few minutes in agreement with the experimental observations.

Potential dependent ion concentration.-It is well known that platinum dissolution under steady state conditions strongly depends on the applied cathode potential. This is reflected by an increase in the platinum ion concentration with increasing potential. To study this dependence we simulate the operation at several constant potentials for $50 \mathrm{~h}$. The temperature was chosen to be $80^{\circ} \mathrm{C}$.

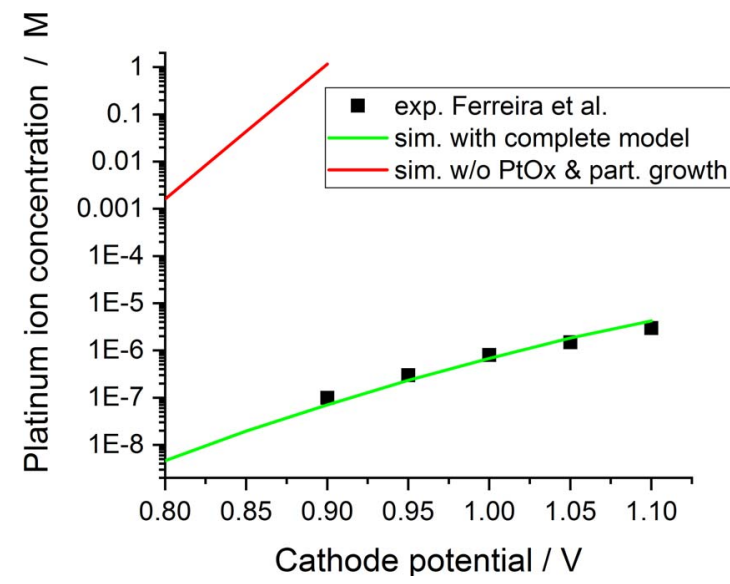

Figure 2. Concentration of $\mathrm{Pt}^{2+}$ depending on the cathode potential. Symbols represent the experimental data taken from Ref. 8, the green line corresponds to the simulation with the complete model while the red line shows the simulated ion concentration at fixed PSD without effect of platinum oxides.

Fig. 2 shows the simulated ion concentration in comparison with experimental values reported in Ref. 8, which have been measured under similar operating conditions. The calculated ion concentrations are in good agreement with the experiments up to about 1.0V. At higher potentials the model slightly over-predicts the ion concentration. This might be due to the simplified platinum oxide model which has been validated only at lower potentials (cf. Platinum oxide coverage section). Note that the increase of ion concentration with potential is significantly lower than what would be expected from a two-electron charge transfer reaction. This is caused by two effects: on the one hand, increasing cathode potential increases the platinum oxide coverage which stabilizes the particles and reduces platinum dissolution; on the other hand, the particle growth during the $50 \mathrm{~h}$ test is enhanced with increasing potential. Since the equilibrium concentration depends on the particle sizes, this also decreases the apparent platinum dissolution rate. For comparison, Fig. 2 also shows the potential dependent ion concentration for fixed PSD without platinum oxide coverage. In this case the ion concentration exceeds the experimental values by several orders of magnitude and the slope is given by the theoretical value $2 \mathrm{~F} /(\mathrm{R} T \ln (10))$. The very high concentrations originate from the presence of very small particles with size $\leq 1 \mathrm{~nm}$ in the pristine PSD. These particles will dissolve very fast which lowers the ion concentration. This demonstrates that the effect of platinum oxides and particle growth are highly important to correctly describe the experimentally observed ion concentrations due to platinum dissolution.

Degradation under AST conditions. - To validate the model with respect to the predicted ECSA loss we simulate the triangle wave (TW) catalyst degradation AST, which consists of fast potential cycling between $0.6 \mathrm{~V}$ and $1.0 \mathrm{~V}$ with a duration of $16 \mathrm{~s}$ per cycle. The operating conditions are similar to the ones used in the corresponding AST experiment (cf. Accelerated Stress Test section). Fig. 3a shows the ECSA evolution during this AST. The model is able to accurately describe the experimentally observed fast drop of ECSA. The inset shows a magnification of the ECSA evolution between cycles 498 and 502. Here, the strong variations of the ECSA loss during the cycles become visible.

To investigate the catalyst degradation during the AST in more detail, Fig. $3 \mathrm{~b}$ shows the evolution of the $\mathrm{Pt}^{2+}$ concentration during cycles 498 to 502 in the AST simulation. As one can see, the ion concentration varies significantly during the potential cycling. At $1.0 \mathrm{~V}$ the concentration is about a factor of 16 higher than in the steady state case (cf. Fig. 2), which is due to the reduction of the platinum oxides during the low potential period. This causes a significant acceleration of the catalyst degradation during the AST. In addition, a second peak in the ion concentration is observed during the sweep to low potentials. This 

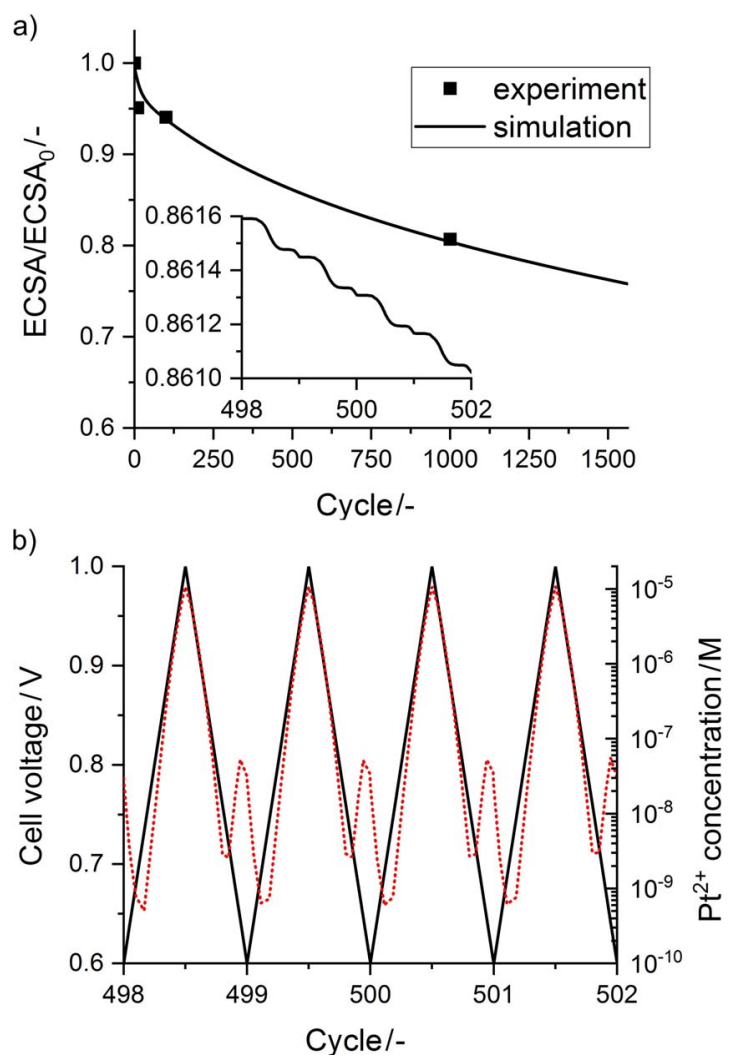

Figure 3. a) ECSA evolution during the TW AST. Symbols represent the measured values the line corresponds to the simulation; b) Simulated average concentration of $\mathrm{Pt}^{2+}$ (dotted red line) in the cathode catalyst layer during AST potential cycling. The cell voltage is shown as black line.

peak is caused by the reduction of the platinum oxides as described by Reaction 11 and also contributes to the accelerated degradation. These two contributions have been also observed experimentally and are discussed in detail in Refs. 10,11.

To evaluate the effect of the AST protocol we also simulate different potential cycles proposed by Harzer et al., ${ }^{9}$ i.e., a triangular wave similar to the previous AST but with upper potential limit of $0.85 \mathrm{~V}$ (TW-LUPL), a square wave between $0.6 \mathrm{~V}$ and $1.0 \mathrm{~V}$ with $8 \mathrm{~s}$ hold at each potential (SW) and a triangular wave with ramp of $50 \mathrm{mV} / \mathrm{s}$ between $0.6 \mathrm{~V}$ and $1.0 \mathrm{~V}$ with $8 \mathrm{~s}$ hold at upper and lower potential (TW-H). These different tests had been designed to distinguish between the effect of holding at high potential and fast potential changes in case of the square wave. Harzer et al. reported that the degradation per cycle in SW and TW-H is quite similar while it is lower for the standard TW AST and significantly lower for the TW-LUPL. Fig. 4 shows the comparison of the different simulated ASTs. Indeed, the obtained trends are the same as observed experimentally. In particular, the ECSA loss per cycle is quite similar for TW-H and SW, even though the duration per cycle is twice in case of the TW-H AST ( 32 seconds). The degradation per cycle of both is about a factor of 2 higher compared to the standard TW AST. Instead, the ECSA loss for the TW-LUPL is by a factor of 5-6 lower compared to the standard TW. These trends are in quite good agreement with the experimental observations in Refs. 9 and 41.

Comparison of catalyst degradation under AST and steady state operation.-In the following we compare the degradation under steady state operation to the one during TW AST operation. This comparison is important since ASTs are often used to test the durability of the MEA. However, in order to be able to conclude about the durability under real operation, one has to ensure that the occurring

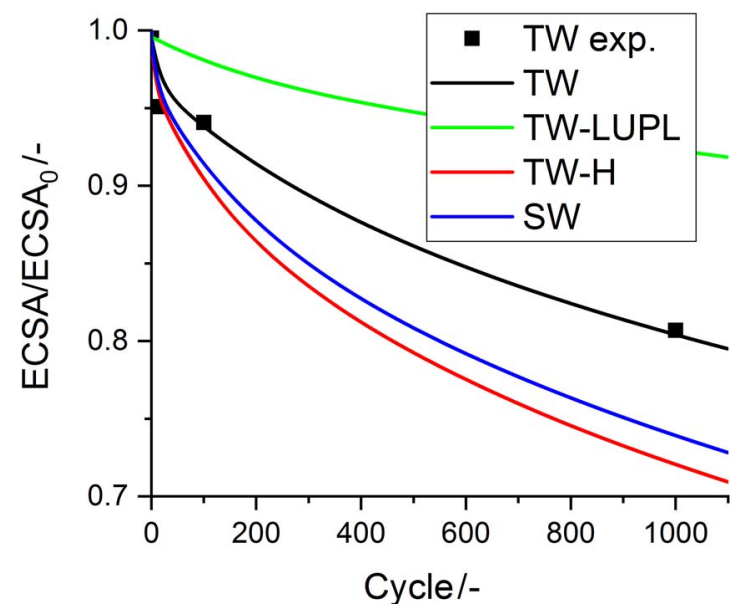

Figure 4. Comparison of ECSA loss for different potential cycles: triangle wave between $0.6 \mathrm{~V}$ and $1.0 \mathrm{~V}$ (TW, black), triangle wave between $0.6 \mathrm{~V}$ and $0.85 \mathrm{~V}$ (TW-LUPL, green), triangle wave between $0.6 \mathrm{~V}$ and $1.0 \mathrm{~V}$ with $8 \mathrm{~s} \mathrm{holds}$ at both potentials (TW-H, red) and square wave between $0.6 \mathrm{~V}$ and $1.0 \mathrm{~V}$ (SW, blue). The sweep rate for all triangle waves is $50 \mathrm{mV} / \mathrm{s}$.

degradation in both cases is similar and, in the ideal case, just scaled with a certain acceleration factor.

Fig. 5a shows the simulated ECSA loss during 2000h of operation at $0.2 \mathrm{~A} \mathrm{~cm}^{-2}$ in air and oxygen at the cathode side. Further operating parameters were temperature $80^{\circ} \mathrm{C}$, relative humidity $100 \%$, pressure
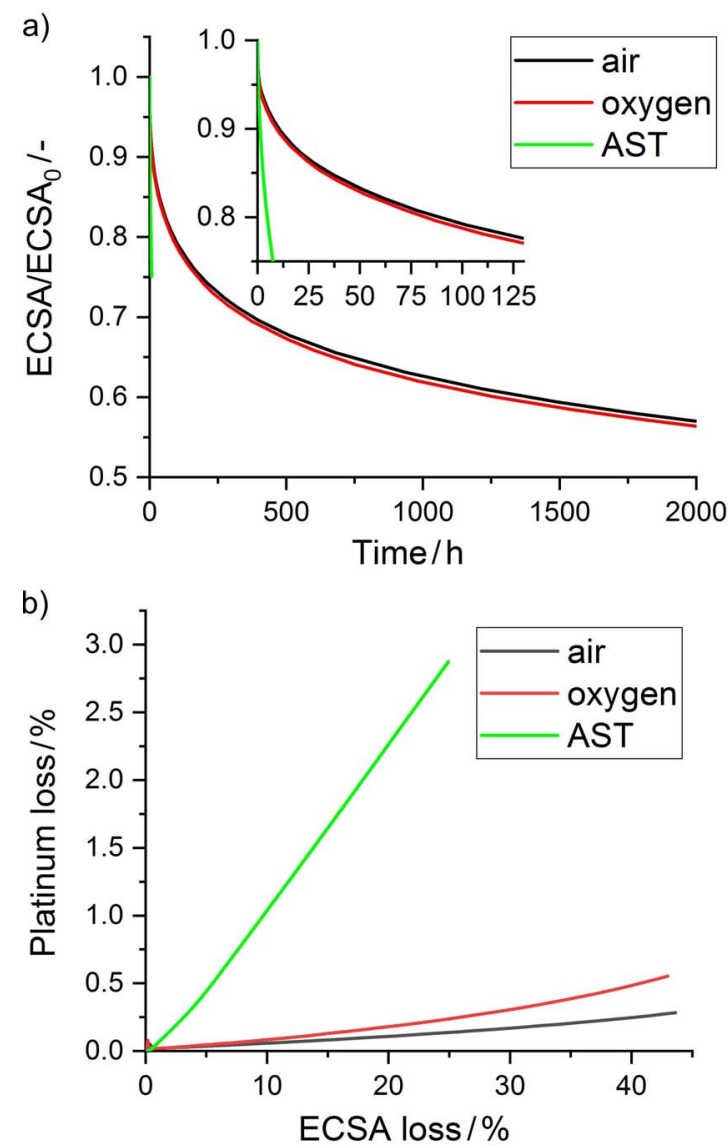

Figure 5. a) Simulated loss of ECSA during steady state operation at $0.2 \mathrm{~A}$ $\mathrm{cm}^{-2}$ in air and pure oxygen compared to AST operation; b) Loss of platinum content in the cathode CL over loss of ECSA for the steady state simulations in air and oxygen compared to the AST. 

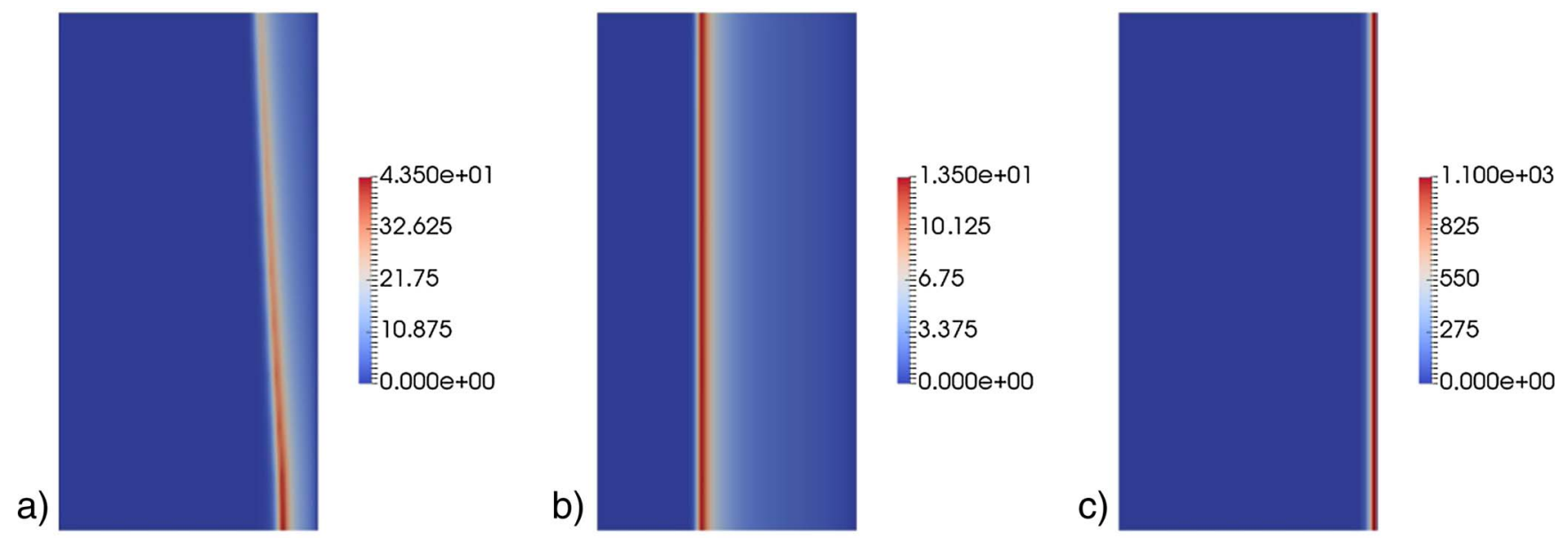

Figure 6. Simulated platinum metal concentration in the membrane in mol $\mathrm{m}^{-3}$ after $25 \%$ ECSA loss for a) operation with air; b) operation with oxygen; c) AST operation. The cathode side is on the right hand side and gas inlet is on the top, respectively. For visualization purposes the figures are scaled by a factor of $10^{4}$ in $\mathrm{x}$-direction.

1.5 bar and stoichiometry 1.5 at anode and 2.0 at cathode, respectively. For comparison the ECSA loss during AST is also included. The ECSA loss in oxygen is slightly higher compared to the operation in air, which can be explained by the higher cathode potential in oxygen. In general, the degradation during steady state operation is significantly lower compared to the AST, which demonstrates the effectiveness of this AST. However, the catalyst degradation under the AST conditions is quite different compared to the steady state cases. In principle the ECSA loss can occur due to the precipitation of platinum from the CL into the membrane, causing a loss of the total platinum amount in the CL or due to Ostwald ripening by which the surface of the particles is reduced while the amount of platinum stays constant. The relation between ECSA loss and platinum loss in the catalyst layer therefore indicates the contribution of both mechanisms to the overall ECSA loss. This relation is shown for the three cases in Fig. 5b. For the AST, the platinum loss in the CL per ECSA loss is significantly higher, which demonstrates that this AST accelerates the the platinum deposition in the membrane more than the particle growth mechanism. This can be attributed to two effects related to the platinum ion transport described by Eq. 21 . On the one hand, the position at which the platinum band is formed is different for these three cases. Fig. 6 shows the simulated platinum distribution in the membrane for both steady-state cases and the AST case. To make them comparable, all images have been taken at the respective points of the degradation tests at which $25 \%$ of ECSA has been lost. As one can see, a well defined platinum band is formed whose position strongly depends on the operating conditions. With air operation the platinum band forms closer to the cathode side while under pure oxygen the platinum band shifts toward the anode. This is because the position of the platinum band depends on the crossover fluxes of oxygen and hydrogen. The platinum band is formed at a position such that the hydrogen flux is twice the oxygen flux, i.e., both fluxes compensate each other at the platinum band due to the hydrogen combustion Reaction 27. Under AST conditions the platinum is deposited directly at the interface between membrane and cathode CL, since no oxygen is present in this case. This generates high concentration gradients for the platinum ions at the membrane-CCL interface, enhancing the platinum band formation. On the other hand, the gradients in ionic potential through the MEA are very low for the AST, while for normal operation these gradients tend to keep the platinum ions on the cathode side. In case of the AST both effects facilitate the transport of platinum ions into the membrane and therefore the platinum band formation. Thus, even though the ECSA loss in all three case is the same at the point shown in Fig. 6, the amount of platinum in the membrane deviates significantly. It is the highest for the AST and the lowest for operation in oxygen.
Finally, we use the model to investigate heterogeneities in the catalyst degradation within the CL. Here, we consider the degradation at the end of the $2000 \mathrm{~h}$ steady state simulation at $0.2 \mathrm{~A} \mathrm{~cm}^{-2}$. Fig. 7 a shows the local platinum density in the CL after the $2000 \mathrm{~h}$ of degradation. In a thin layer close to the membrane the platinum density is significantly reduced, since platinum has precipitated into the membrane. On the other hand, particle growth is quite homogeneous through the thickness of the CL except for the thin depletion zone close to the membrane where particle growth is hindered. Fig. $7 \mathrm{~b}$ shows the distribution of the average particle size in the CL while Fig. 7c shows the local particle size distributions at four distinct points of the CL: at the membrane side and GDL side close to the gas inlet as well as at the gas outlet, respectively. While the particle growth is significantly reduced in the depletion zone at the membrane side, the distributions are quite homogeneous along the channel. Note that different observations have been made experimentally regarding the heterogeneity of particle growth in literature. While some authors report larger particle sizes on the GDL side ( $\mathrm{cf}^{42}$ ) others observe the opposite trend. ${ }^{8}$ This indicates that these trends are dependent on the materials or operating conditions. Indeed, with our model we observe that the position of the largest particle growth within the CL depends on the transport parameters in the CL, such as the ionic conductivity and oxygen transport resistance in the agglomerate. Those parameters affect the current density distribution in the CL and the local degradation rates. The trends shown in this work are obtained for the parametrization of the single cell model given in Ref. 26.

It is worth mentioning that the enhanced platinum deposition in the membrane during the AST discussed before (Fig. 5b) also enhances the formation of the depletion zone close to the membrane and therefore will cause higher performance losses per total ECSA loss compared to aging under normal operation. The different aging behavior suggests that the degradation observed under AST conditions cannot directly be used as indication for the degradation under real operation. Therefore, the development of new ASTs in which degradation is closer to the one under real operation is desirable.

ECSA loss and particle growth in Direct Methanol Fuel Cell.The model has also been applied to simulate the catalyst degradation in the Direct Methanol Fuel Cell (DMFC) degradation test described in the DMFC stress test section. Since the same catalyst has been used as in the PEMFC case, all of the modeling parameters describing the degradation mechanism are the same. However, some modifications had to be done to simulate the DMFC degradation test as described in the following. Using reference electrodes it has been shown that the cathode potential in DMFC is typically around $0.85 \mathrm{~V}$, independent on the current density. ${ }^{43}$ Therefore, in order to simulate the degradation 
a)

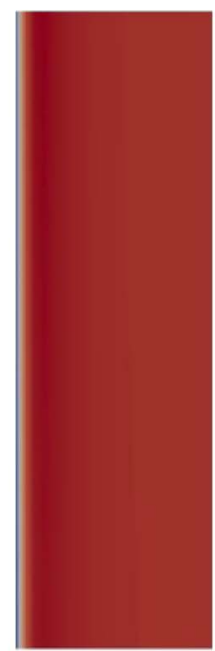

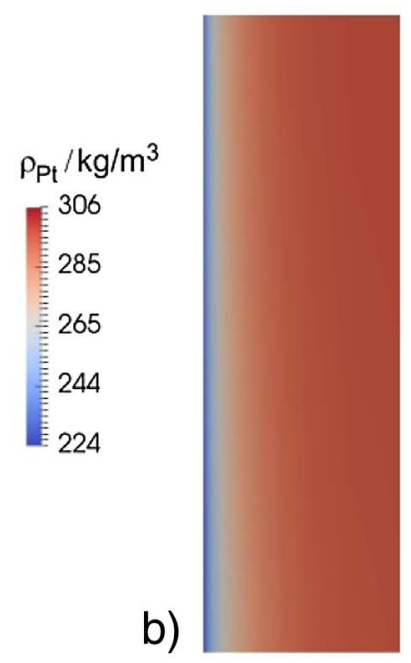
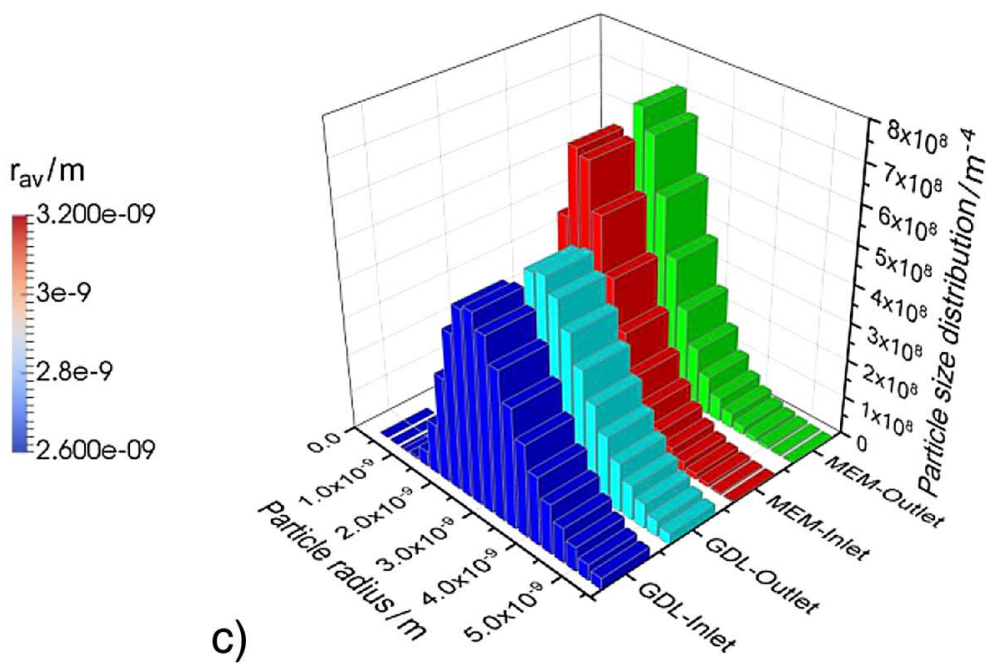

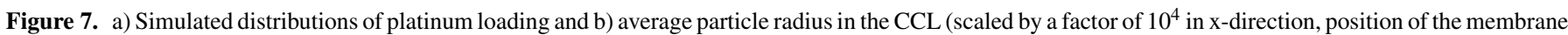
on the left) as well as c) local particle size distribution at four positions of the CCL after $2000 \mathrm{~h}$ at $0.2 \mathrm{~A} \mathrm{~cm}-2$ with air at cathode side.

during the 500h aging test in DMFC, we set the cathode potential to $0.85 \mathrm{~V}$. The relative humidity is taken to be 100 percent due to the high water content in DMFC. In DMFC operation a refresh procedure is performed every 20 minutes to recover reversible degradation. ${ }^{38}$ During this refresh, the cathode potential drops to low values which leads to a reduction of the platinum oxides. To simplify the simulation we do not resolve all these complex refresh procedures during the $500 \mathrm{~h}$ test but instead consider an average fixed platinum oxide coverage of $\theta_{\mathrm{PtO}}=0.1523$, which corresponds to the calculated value 5 minutes after the refresh. In this way we imitate the effect of the periodic refresh during the test on the otherwise logarithmically increasing oxide coverage. Additionally, the platinum band formation sub-model has been deactivated for the DMFC case since in DMFC no hydrogen is present which is needed for the platinum band formation according to Eq. 25. The absence of a platinum band in DMFC has also been confirmed experimentally by TEM observations of the ionomer membrane in the aged DMFC MEA. ${ }^{37}$

Two different measures are used for model validation in this case: the ECSA evolution during the test and the change of the PSD. In order to be able to compare experiment and simulation in this case, it important to also take into account the activation period before the degradation test, since the initial PSD has been measured in a pristine MEA before activation and therefore does not correspond to the PSD at the beginning of the degradation test. We model the activation period by an additional operation at $0.85 \mathrm{~V}$ for 6 hours before starting the 500h degradation test. As shown in Fig. 8a, the simulation predicts a significant effect of this short activation period on the PSD. The reason for this are the very small particles of $\leq 1 \mathrm{~nm}$ in the pristine MEA which dissolve very fast. Taking into account this initial activation period, the model accurately describes the ECSA evolution shown in Fig. $8 \mathrm{~b}$ during the $500 \mathrm{~h}$ degradation test as well as the PSD which has been measured at the end of test in Fig. 8a. The degradation might be slightly underestimated due to neglecting the refresh procedures which might lead to some small additional dissolution related to the oxide reduction.

\section{Conclusions}

We have developed a multiscale catalyst degradation model for low temperature fuel cells which includes oxidation and reduction of the platinum nanoparticles, platinum dissolution, particle growth, platinum ion transport and platinum band formation. The model has been coupled to a 2D single cell model in order to simulate the spatially resolved catalyst degradation under various operating conditions, including accelerated stress tests. The model has been validated with respect to potential dependent platinum oxide coverages and ion concentrations, particle size distribution and ECSA loss for an AST as well as under DMFC operation.

The accuracy of the model is demonstrated by correctly describing the following experimental observations:

a)

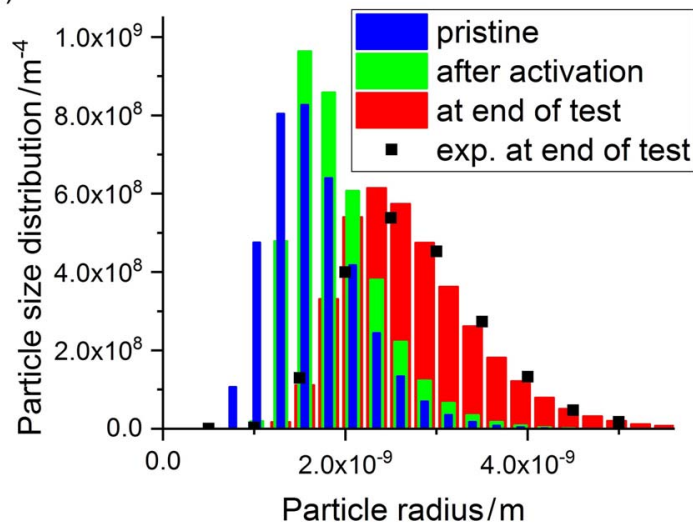

b)

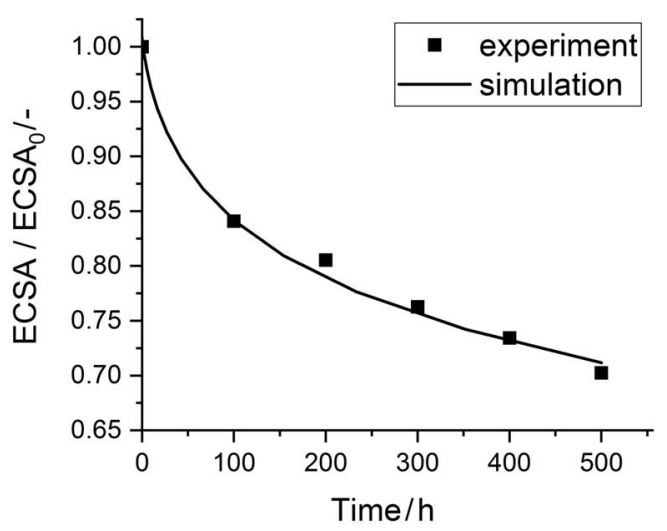

Figure 8. a) Evolution of the PSD during DMFC degradation test: initial PSD of the pristine cell (blue), simulated PSD after activation (green), simulated PSD after degradation test (red) and measured PSD after degradation test (symbols); b) Comparison of simulated and measured evolution of the ECSA during DMFC degradation test. 
1. The platinum oxide coverage strongly depends on the cathode potential and increases logarithmically in time. This time-evolution can be described by the semi-empirical three-step mechanism proposed in this paper.

2. Periodic reduction of platinum oxides and the consequent lowering of surface tension cause the accelerated catalyst degradation during potential cycling.

3. With a single set of parameters the model provides accurate results regarding potential dependent platinum ion concentration, particle growth and ECSA loss under various operating conditions, confirming the reliability of the model even under transient operation.

4. The position of the platinum band and amount of deposited platinum in membrane strongly depend on the operating conditions, especially on the gas composition at the cathode.

5. The precipitation of platinum into the membrane creates a depletion zone at the membrane side of the catalyst layer with a lower ECSA and reduced platinum particle growth.

6. Comparison of different potential cycles shows the highest ECSA loss per time for the square wave cycle, while the degradation per cycle for square wave with $16 \mathrm{~s}$ period and triangle wave with potential holding with $32 \mathrm{~s}$ period was quite similar.

Furthermore, based on the simulation results two important conclusions can be made with respect to the analysis of catalyst degradation:

1. The standard catalyst ASTs consisting of potential cycles with nitrogen at the cathode accelerate the platinum precipitation into the membrane more than the Ostwald-ripening. This is reflected by a higher platinum loss in the CL per ECSA loss compared to longterm, steady-state degradation tests. The higher precipitation in case of the ASTs leads to more pronounced depletion zone close to the membrane which will lead to a different performance degradation. The development of advanced ASTs is therefore needed to obtain a degradation behavior which is more representative of real aging.

2. Analysis of both PSD and ECSA evolution is important to distinguish the different catalyst degradation mechanisms. The activation period before the actual degradation tests can significantly affect the PSD especially if very small particles with radius $\leq 1 \mathrm{~nm}$ are present in the initial PSD. Therefore, a measurement of the PSD after activation provides a better characterization of the fresh cell compared to the pristine PSD. Otherwise, inconsistencies between measured PSD and ECSA at beginning of test might occur.

\section{Acknowledgments}

The authors thank the Dumu ${ }^{\mathrm{X}}$ developers at the IWS - Department of Hydromechanics and Modelling of Hydrosystems, University of Stuttgart, for their excellent work and kind support. We also thank Laure Guetaz from CEA for the TEM analysis. The research leading to these results has received funding from the European Union's Seventh Framework Program (FP7/2007-2013) for the Fuel Cells and Hydrogen Joint Technology Initiative under grant agreement $n^{\circ} .621216$ and from the European Union's Horizon 2020 research and innovation program under grant agreement $\mathrm{n}^{\circ} .779565$.

\section{Appendix A: Surface Tension}

In the following we derive Equation 13 for the surface tension of a platinum particle with oxide coverage. We start from the Gibbs fundamental equation

$$
d G=-S d T+V d P+\sum_{i} \mu_{i} d n_{i}+\gamma d A
$$

For constant temperature, pressure and surface area A1 simplifies to

$$
d G=\sum_{i} \mu_{i} d n_{i}
$$

We consider a reaction of the type

$$
\mathrm{A}+(\mathrm{S}) \leftrightarrow \mathrm{B}(\mathrm{S})+\mathrm{C}
$$

which can be described by the following generalized Frumkin isotherm kinetics

$$
r=k_{f} a(1-N \theta) e^{-\frac{\omega \theta}{\mathrm{R} T}}-k_{b} \theta
$$

where $\theta$ is the surface coverage. By introducing the stoichiometric numbers $v_{i}$ we can rewrite

$$
n_{i}=A \Gamma \nu_{i} \theta
$$

where $\Gamma$ is the number of sites per surface area. Thus, we have

$$
d G=\sum_{i} \mu_{i} d n_{i}=A \Gamma \sum_{i} \nu_{i} \mu_{i} d \theta=A \Gamma \Delta_{r} G d \theta
$$

Therefore, the Gibbs free energy of the particle can be calculated as

$$
G(T, p, n)=G^{0}(T, p)+A \gamma_{\text {pure }}+A \Gamma \int_{0}^{\theta} \Delta_{r} G\left(\theta^{\prime}\right) d \theta^{\prime} .
$$

This leads to the coverage dependent surface tension

$$
\gamma(\theta)=\left(\frac{\partial G}{\partial A}\right)_{T, p, n}=\gamma_{\text {pure }}+\Gamma \int_{0}^{\theta} \Delta_{r} G\left(\theta^{\prime}\right) d \theta^{\prime} .
$$

By rewriting

$$
\begin{aligned}
\Delta_{r} G & =\sum_{i} v_{i} \mu_{i}=\sum_{i} v_{i} \mu_{i}^{0}+v_{i} \mathrm{R} T \ln \left(a_{i}\right) \\
& =\Delta_{r} G^{0}+\mathrm{R} T \sum_{i} v_{i} \ln \left(a_{i}\right)=\mathrm{R} T\left[-\ln K+\ln \left(\prod_{i} a_{i}^{v_{i}}\right)\right] \\
& =\mathrm{R} T \ln \left(\frac{r_{\mathrm{rev}}}{r_{\mathrm{fwd}}}\right)
\end{aligned}
$$

the surface tension can also be written by means of the surface reaction kinetics as

$$
\gamma(\theta)=\gamma_{0}+\Gamma \mathrm{R} T \int_{0}^{\theta} \ln \left(\frac{r_{\mathrm{rev}}\left(\theta^{\prime}\right)}{r_{\mathrm{fwd}}\left(\theta^{\prime}\right)}\right) d \theta^{\prime} .
$$

For the reaction kinetics defined in Eq. A4 we obtain

$$
\begin{aligned}
\gamma(\theta) & =\gamma_{0}+\Gamma \mathrm{R} T \int_{0}^{\theta} \ln \left(\frac{k_{b} \theta^{\prime}}{k_{f} e^{-\frac{\omega \theta^{\prime}}{\mathrm{R} T}}\left(1-N \theta^{\prime}\right) a}\right) d \theta^{\prime} \\
& =\gamma_{0}+\Gamma \mathrm{R} T\left[\theta \ln (\theta)+\left(\frac{1}{N}-\theta\right) \ln (1-N \theta)+\ln \left(\frac{k_{b}}{k_{f} a}\right) \theta+\frac{\omega \theta^{2}}{2 \mathrm{R} T}\right]
\end{aligned}
$$

For the platinum oxide kinetics defined in Eqs. 4-6 this finally leads to the surface tension in Equation 13.

\section{List of Symbols}

$A(r) \quad$ Surface area of all particles with radius $r$ per catalyst layer volume $/ \mathrm{m}^{2} \mathrm{~m}^{-3}$

$a_{i} \quad$ activity of species $i /-$

$c_{i} \quad$ concentration of species $i / \mathrm{mol} \mathrm{m}^{-3}$

ECSA electrochemically active surface area $/ \mathrm{m}^{2} \mathrm{~m}^{-3}$

$E_{i} \quad$ equilibrium potential of reaction $i / \mathrm{V}$

$\mathrm{F} \quad$ Faraday constant/ $\mathrm{C} \mathrm{mol} \mathrm{m}^{-1}$

$i \quad$ current density/A $\mathrm{m}^{2}$

$k_{f} \quad$ forward rate constant of platinum dissolution reaction $/ \mathrm{s}^{-1}$

$k_{i} \quad$ rate constant of platinum oxide reaction $i / \mathrm{s}^{-1}$

$k_{\mathrm{Pt} \text {-band }}$ rate constant of platinum band formation $/ \mathrm{m}^{3} \mathrm{~mol}^{-1} \mathrm{~s}^{-1}$

$k_{\mathrm{H} 2 \mathrm{c}}$ rate constant of hydrogen combustion reaction $/ \mathrm{m}^{9} \mathrm{~mol}^{-3}$ $\mathrm{s}^{-1}$

$k_{r} \quad$ backward rate constant of platinum dissolution reaction $/ \mathrm{m}^{3}$ $\mathrm{mol}^{-1} \mathrm{~s}^{-1}$

$M_{\mathrm{Pt}} \quad$ platinum loading $/ \mathrm{kg} \mathrm{m}^{-2}$

$N(r) \quad$ particle size distribution $/ \mathrm{m}^{-4}$

$N_{0} \quad$ Scaling factor in particle size distribution/-

$n_{\text {edge }} \quad$ number of platinum atoms along the particle edge/-

$n_{i} \quad$ number of electrons involved in reaction $i /$ -

$q_{i} \quad$ source term for species $i / \mathrm{C} \mathrm{m}^{-3}$ or $\mathrm{mol} \mathrm{m}^{-3}$

$\mathrm{R} \quad$ universal gas constant/J $\mathrm{mol}^{-1} \mathrm{~K}^{-1}$

$r_{i} \quad$ volumetric reaction rate of platinum oxide reaction $i / \mathrm{s}^{-1}$

$r_{P t, i} \quad$ volumetric reaction rate of dissolution reaction $i / \mathrm{mol} \mathrm{m}^{-3}$ $\mathrm{s}^{-1}$ 
$r_{\mathrm{Pt}-\text { band }} \quad$ volumetric reaction rate of platinum band formation reaction $/ \mathrm{mol} \mathrm{m}^{-3} \mathrm{~s}^{-1}$

$r_{\mathrm{H} 2 \mathrm{c}} \quad$ volumetric reaction rate of hydrogen combustion reaction $i / \mathrm{mol} \mathrm{m}^{-3} \mathrm{~s}^{-1}$

$T \quad$ temperature/K

$t \quad$ time/s

$u_{\mathrm{Pt}^{2+}} \quad$ mobility of platinum ions in ionomer/s mol kg-1

$X_{\text {edge }} \quad$ fraction of edge sites/-

\section{Greek}

$\alpha_{i} \quad$ transfer coefficient of reaction $i /-$

$\Gamma \quad$ site density of platinum $/ \mathrm{mol} \mathrm{m}^{-2}$

$\gamma \quad$ surface tension $/ \mathrm{N} \mathrm{m}^{-1}$

$\gamma_{0} \quad$ surface tension of $\mathrm{Pt}[111] / \mathrm{N} \mathrm{m}^{-1}$

$\Delta \phi \quad$ electrode potential/V

$\theta_{i} \quad$ coverage of surface species $i /$ -

$\mu \quad$ chemical potential/J $\mathrm{mol}^{-1}$

$\phi_{\text {ionomer }}$ ionomer potential/V

$\Omega \quad$ molar volume of platinum $/ \mathrm{m}^{3} \mathrm{~mol}^{-1}$

$\omega_{i} \quad$ interaction parameter of species $i / \mathrm{J} \mathrm{mol}^{-1}$

$\zeta \quad$ catalyst utilization/-

\section{ORCID}

\section{Thomas Jahnke (D) https://orcid.org/0000-0003-2286-6801}

\section{References}

1. Toyota. https://global.toyota/en/detail/4198334/.

2. Honda. https://automobiles.honda.com/clarity-fuel-cell.

3. Hyundai. https://www.hyundainews.com/models/hyundai-nexo-2019-nexo

4. Daimler. https://www.daimler.com/products/passenger-cars/mercedes-benz/glc-fcell.html.

5. M. Eikerling and A. Kulikovsky, "Polymer Electrolyte Fuel Cells," CRC Press, 2014

6. J. Wu, X. Z. Yuan, J. J. Martin, H. Wang, J. Zhang, J. Shen, S. Wu, and W. Merida, “A review of PEM fuel cell durability: Degradation mechanisms and mitigation strategies," Journal of Power Sources, 184(1), 104 (2008).

7. Y. Shao-Horn, W. C. Sheng, S. Chen, P. J. Ferreira, E. F. Holby, and D. Morgan, "Instability of supported platinum nanoparticles in low-temperature fuel cells," Topics in Catalysis, 46(3-4), 285 (2007).

8. P. J. Ferreira, G. J. la O', Y. Shao-Horn, D. Morgan, R. Makharia, S. Kocha, and H. A. Gasteiger, "Instability of ptc electrocatalysts in proton exchange membrane fuel cells," Journal of The Electrochemical Society, 152(11), A2256 (2005).

9. G. S. Harzer, J. N. Schwämmlein, A. M. Damjanović, S. Ghosh, and H. A. Gasteiger, "Cathode loading impact on voltage cycling induced PEMFC degradation: A voltage loss analysis," Journal of The Electrochemical Society, 165(6), F3118 (2018).

10. A. A. Topalov, S. Cherevko, A. R. Zeradjanin, J. C. Meier, I. Katsounaros, and K. J. J. Mayrhofer, "Towards a comprehensive understanding of platinum dissolution in acidic media," Chem. Sci., 5(2), 631 (2014).

11. S. Cherevko, G. P. Keeley, S. Geiger, A. R. Zeradjanin, N. Hodnik, N. Kulyk, and K. J. J. Mayrhofer, "Dissolution of platinum in the operational range of fuel cells," ChemElectroChem, 2(10), 1471 (2015).

12. R. M. Darling, J. P. Meyers, and Kinetic model of platinum dissolution in, PEMFCs," Journal of The Electrochemical Society, 150(11), A1523 (2003).

13. W. Bi, G. E. Gray, and T. F. Fuller, "PEM fuel cell ptc dissolution and deposition in nafion electrolyte," Electrochemical and Solid-State Letters, 10(5), B101 (2007).

14. T. Jahnke, G. Futter, A. Latz, T. Malkow, G. Papakonstantinou, G. Tsotridis, P. Schott, M. Gérard, M. Quinaud, M. Quiroga, A. Franco, K. Malek, F. Calle-Vallejo, R. F. de Morais, T. Kerber, P. Sautet, D. Loffreda, S. Strahl, M. Serra, P. Polverino, C. Pianese, M. Mayur, W. Bessler, and C. Kompis, "Performance and degradation of proton exchange membrane fuel cells: State of the art in modeling from atomistic to system scale," Journal of Power Sources, 304, 207 (2016).

15. R. M. Darling and J. P. Meyers, "Mathematical model of platinum movement in PEM fuel cells," Journal of The Electrochemical Society, 152(1), A242 (2005).

16. E. F. Holby, W. Sheng, Y. Shao-Horn, and D. Morgan, "Pt nanoparticle stability in PEM fuel cells: influence of particle size distribution and crossover hydrogen," Energy \& Environmental Science, 2(8), 865 (2009)

17. E. F. Holby and D. Morgan, "Application of pt nanoparticle dissolution and oxidation modeling to understanding degradation in PEM fuel cells," Journal of The Electrochemical Society, 159(5), B578 (2012).
18. S. G. Rinaldo, J. Stumper, and M. Eikerling, "Physical theory of platinum nanoparticle dissolution in polymer electrolyte fuel cells," The Journal of Physical Chemistry C, 114(13), 5773 (2010).

19. S. F. Burlatsky, M. Gummalla, V. V. Atrazhev, D. V. Dmitriev, N. Y. Kuzminyh, and N. S. Erikhman, "The dynamics of platinum precipitation in an ion exchange membrane," Journal of The Electrochemical Society, 158(3), B322 (2011).

20. R. K. Ahluwalia, S. Arisetty, X. Wang, X. Wang, R. Subbaraman, S. C. Ball, S. DeCrane, and D. J. Myers, "Thermodynamics and kinetics of platinum dissolution from carbon-supported electrocatalysts in aqueous media under potentiostatic and potentiodynamic conditions," Journal of The Electrochemical Society, 160(4), F447 (2013).

21. R. K. Ahluwalia, S. Arisetty, J.-K. Peng, R. Subbaraman, X. Wang, N. Kariuki, D. J. Myers, R. Mukundan, R. Borup, and O. Polevaya, "Dynamics of particle growth and electrochemical surface area loss due to platinum dissolution," Journal of The Electrochemical Society, 161(3), F291 (2014).

22. H. A. Baroody, D. B. Stolar, and M. H. Eikerling, "Modelling-based data treatment and analytics of catalyst degradation in polymer electrolyte fuel cells," Electrochimica Acta, 283, 1006 (2018).

23. P. Urchaga, T. Kadyk, S. G. Rinaldo, A. O. Pistono, J. Hu, W. Lee, C. Richards, M. H. Eikerling, and C. A. Rice, "Catalyst degradation in fuel cell electrodes: Accelerated stress tests and model-based analysis," Electrochimica Acta, 176, 1500 (2015).

24. B. Jayasankar and K. Karan, "O2 electrochemistry on pt: A unified multi-step model for oxygen reduction and oxide growth," Electrochimica Acta, 273, 367 (2018).

25. P. Schneider, C. Sadeler, A.-C. Scherzer, N. Zamel, and D. Gerteisen, "Fast and reliable state-of-health model of a PEM cathode catalyst layer," Journal of The Electrochemical Society, 166(4), F322 (2019).

26. G. A. Futter, P. Gazdzicki, K. A. Friedrich, A. Latz, and T. Jahnke, "Physical modeling of polymer-electrolyte membrane fuel cells: Understanding water management and impedance spectra," Journal of Power Sources, 391, 148 (2018).

27. G. A. Futter, A. Latz, and T. Jahnke, "Physical modeling of chemical membrane degradation in polymer electrolyte membrane fuel cells: Influence of pressure, relative humidity and cell voltage," Journal of Power Sources, 410-411, 78 (2019).

28. B. E. Conway, B. Barnett, H. Angerstein-Kozlowska, and B. V. Tilak, "A surfaceelectrochemical basis for the direct logarithmic growth law for initial stages of extension of anodic oxide films formed at noble metals," The Journal of Chemical Physics, 93(11), 8361 (1990).

29. E. L. Redmond, B. P. Setzler, F. M. Alamgir, and T. F. Fuller, "Elucidating the oxide growth mechanism on platinum at the cathode in PEM fuel cells," Physical Chemistry Chemical Physics, 16(11), 5301 (2014).

30. L. Favre, V. Dupuis, E. Bernstein, P. Mélinon, A. Pérez, S. Stanescu, T. Epicier, J.-P. Simon, D. Babonneau, J.-M. Tonnerre, and J.-L. Hodeau, "Structural and magnetic properties ofCoPtmixed clusters," Physical Review B, 74(1) (2006).

31. P. Parthasarathy and A. V. Virkar, "Electrochemical ostwald ripening of pt and ag catalysts supported on carbon," Journal of Power Sources, 234, 82 (2013).

32. W. Bi, Q. Sun, Y. Deng, and T. F. Fuller, "The effect of humidity and oxygen partial pressure on degradation of pt/c catalyst in PEM fuel cell," Electrochimica Acta, 54(6), 1826 (2009).

33. S. Helmly, B. Ohnmacht, P. Gazdzicki, R. Hiesgen, E. Glzow, and K. A. Friedrich, "Influence of the distribution of platinum deposits on the properties and degradation of platinum-impregnated nafion membranes," Journal of The Electrochemical Society, 161(14), F1416 (2014)

34. A. Z. Weber and J. Newman, "Transport in polymer-electrolyte membranes," Journal of The Electrochemical Society, 151(2), A311 (2004).

35. B. Flemisch, M. Darcis, K. Erbertseder, B. Faigle, A. Lauser, K. Mosthaf, S. Müthing, P. Nuske, A. Tatomir, M. Wolff, and R. Helmig, "DuMux: DUNE for multi\{phase,component,scale,physics,...\} flow and transport in porous media," Advances in Water Resources, 34(9), 1102 (2011).

36. N. Garland, T. Benjamin, and J. Kopasz, "DOE fuel cell program: Durability technical targets and testing protocols, ECS Transactions, 923 (2007).

37. C. Rabissi, P. Gazdzicki, L. Guétaz, S. Escribano, L. Grahl-Madsen, A. Baricci, and A. Casalegno, "A locally resolved investigation on direct methanol fuel cell uneven components fading: Steady state and degradation local analysis," Journal of Power Sources, 397, 361 (2018).

38. C. Rabissi, E. Brightman, G. Hinds, and A. Casalegno, "In operando measurement of localised cathode potential to mitigate DMFC temporary degradation," International Journal of Hydrogen Energy, 43(20), 9797 (2018).

39. H. Yu, A. Baricci, A. Casalegno, L. Guetaz, L. Bonville, and R. Maric, "Strategies to mitigate pt dissolution in low pt loading proton exchange membrane fuel cell: II. a gradient pt loading design," Electrochimica Acta, 247, 1169 (2017).

40. T. Nagai, H. Murata, and Y. Morimoto, "Analysis of the relation between oxidation state and ORR activity of pt by linear sweep voltammetry, in: ECS Transactions, ECS, 2010, pp. 125-130.

41. A. Kneer, N. Wagner, C. Sadeler, A.-C. Scherzer, and D. Gerteisen, "Effect of dwell time and scan rate during voltage cycling on catalyst degradation in PEM fuel cells," Journal of The Electrochemical Society, 165(10), F805 (2018).

42. M. Mench, E. Kumbur, T. Veziroglu, and T. Veziroğlu, "Polymer Electrolyte Fuel Cell Degradation, Elsevier Science, 2012. https://books.google.de/books?id= n54R4M7SMsIC.

43. M. Zago, A. Bisello, A. Baricci, C. Rabissi, E. Brightman, G. Hinds, and A. Casalegno, "On the actual cathode mixed potential in direct methanol fuel cells," Journal of Power Sources, 325, 714 (2016). 